\title{
Modeling and Simulating of Network Public Opinion Evolution Based on Dynamic Reference Point of Prospect Theory
}

\author{
Jianhua Dai, a, Yanan $\mathrm{Li}^{2, \mathrm{~b}}$ \\ ${ }^{1}$ School of Economics \& Management, Nanjing University of Science \& Technology, Nanjing, \\ 210094, China \\ ${ }^{2}$ School of Economics \& Management, Nanjing University of Science \& Technology, Nanjing, \\ 210094, China \\ adjh705@163.com, bliyanan1170105@163.com
}

\begin{abstract}
Keywords: Dynamic Reference Point. Prospect Theory. Network Public Opinion. Evolution.
Abstract. In order to better regulate the cyberspace and guide network public opinion in a right direction, forecasting the trend of network public opinion evolution has been a hotspot in recent years. Generally, netizens are often vulnerable to external factors, but more are affected by "bounded rationality" from the internal. Especially in emergencies, netizens often express their opinions on the basis of their own habits, intuition or experiences rather than searching enough information for decision-making. However, most existing quantitative analysis related to public opinion evolution focus on complete rationality of human, assuming that netizens are rational, intelligent, etc. to make perfect decisions, but it is inconsistent with the reality. Thus we introduced the dynamic reference point of Prospect Theory for constructing the modified HK model, aiming to analyze the migration of psychological reference point in the evolution of network public opinion. Compared with current common models, the proposed model, due to considering the dynamic psychological reference point under bounded rationality, is more realistic in the public opinion evolution.
\end{abstract}

\section{Introduction}

According to the $39^{\text {th }}$ China Statistical Report on Internet Development from CNNIC, the number of Chinese netizens has reached 731 million that is equivalent to the total population of Europe up to December 2016 [1]. Moreover, as social media like WeChat, Microblog, Quora, etc., have been increasingly popular and the Internet has been continuously developing in China recently, lowered threshold of network information release allows netizens to share experience and express opinions openly and freely. However, various network information contents are often filled with reaction, violence, superstition, etc., and Internet applications are provided with characteristics such as virtuality, concealment, arbitrariness, etc. Furthermore, netizens interpret information and make choices with bounded rationality, then their emotions and opinions are scattered into collision fusion, easily causing network public opinion, then affecting social stability and harmony. Therefore, the research on how to quickly and effectively quell various public opinion events, reflecting authority and credibility of the government, has become important theoretically and practically.

Throughout studies on network public opinion, more qualitative analyses of its formation [2, 3], features $[4,5]$, and influencing factors [6] have existed, but qualitative analysis, to some degree, is subjective, and also difficult to reflect local differences between things. In recent years, studies on the micro-level public opinion evolution have emerged in an endless stream [7, 8, 9], where quantitative models that simulate reality on simulation platforms have played a crucial role in the supervision and guidance of network public opinion. Most researches assumed that netizens were completely rational, that is, they could pursue optimal decision-making. However, Herbert Simon, Nobel laureate, pointed out that it's difficult to acquire utility or profit maximization because of individual bounded rationality [10]. Under network public opinion, netizens have not enough time and energy to search for entire information and verify authenticity, and importantly each person is limited by knowledge, experience and ability. So when facing all kinds of uncertainties on the Internet, netizens would acquire a limited rational decision-making by virtue of their habits, intuition, experience, etc. Thus, despite the fact that models constructed by existing quantitative analysis are "perfect" on simulation platforms, it is not necessary for netizens to act as "rules" of the proposed models. In a word, the 
current difficulty on network public opinion evolution lies in how to introduce psychological dynamics of netizens into the evolution that is also the problem we intend to solve at present.

At the beginning of an incident outbreak, although netizens did not grasp all information, they still held initial opinions. As time goes by, netizens, affected by social circle positively or negatively, would change their opinions in varying degrees, then the opinion deviates from the original one. Actually, each person expects that the incident develops in accordance with his judgments, but his expectation would transform owing to the interaction with neighbors and mastering latest messages. This is dynamic psychological expectation (i.e. dynamic reference point). Back in 1979, Kahneman and Tversky studied the reference point transfer, and they proposed Prospect Theory that described human decision-making process $[11,12]$. The reference point in Prospect Theory determines the outcome is gain or loss from individual, and then affects human preferences and decision-making [13]. Studies on the reference point are quite abundant currently, such as determinants [14], classification [15], application in other disciplines [16, 17, 18], etc., but most of them concentrated on static reference point. Though some researches involved dynamic reference point, for example, Wang et al. proposed a prospect theory-based interval dynamic reference point method for emergency decision making to response to emergency events such as terrorist attacks or natural disasters immediately [16], similar studies are still few, and none considered dynamic reference point in public opinion evolution. As a consequence, considering human's bounded rationality, we apply dynamic reference point from Prospect Theory to examine how individual psychological expectation transfers and influences the evolution of network public opinion. Then, we construct a new model that adds dynamic reference point to Hegselmann-Krause model to explore the evolving process.

The paper is organized as follows. First, we introduce Prospect Theory and dynamic reference point briefly. After the proposed model is described, we then discuss the results of the simulation on the Netlogo platform. The final section offers conclusion.

\section{Prospect Theory}

Model Description. In the 1970s, Kahneman and Tversky first proposed Prospect Theory (PT) [11]. In 1992, both improved the theory further that described human decision-making behavior under risk and uncertainty in the form of formulas [12]. It is precisely because 1) PT models two major deviations from expected utility: nonlinear decision weighting and loss aversion, i.e., the tendency that people treat outcomes as deviations from a reference point and are more sensitive to losses than to gains of the same magnitude $[19,20]$; and 2) the formulas have the characteristics of a simple computation process and clear logic, PT has been used widely to solve decision-making problems considering decision makers' psychological behavior.

PT distinguishes two phases in the choices process: an early phase of editing and a subsequent phase of evaluation [11]. In the editing phase, outcomes are reflected gains or losses by reference point. In the evaluation phase, the edited prospects are evaluated by a value function and a weighting function. The value function measures the value of deviations from the reference point, as expressed in the form of power law as Eq. 1.

$$
v(\Delta x)=\left\{\begin{array}{ll}
\Delta x^{\alpha} & \text { if } \Delta x \geq 0 \\
-\lambda(-\Delta x)^{\beta} & \text { if } \Delta x<0
\end{array} .\right.
$$

In Eq. 1, $\Delta x$ denotes the gains or losses; $\Delta x \geq 0$ represents gains and $\Delta x<0$ represents losses. $\alpha$ and $\beta$ are exponent parameters related to gains and losses, respectively, $0 \leq \alpha, \beta \leq 1$. $\lambda$ is the risk aversion parameter, representing the characteristic of steeper for losses than for gains, $\lambda>1$. Fig. 1 shows the value function with convex and concave $S$-shapes. The values of $\alpha, \beta$ and $\lambda$ are generally determined through experiments $[12,19]$. 


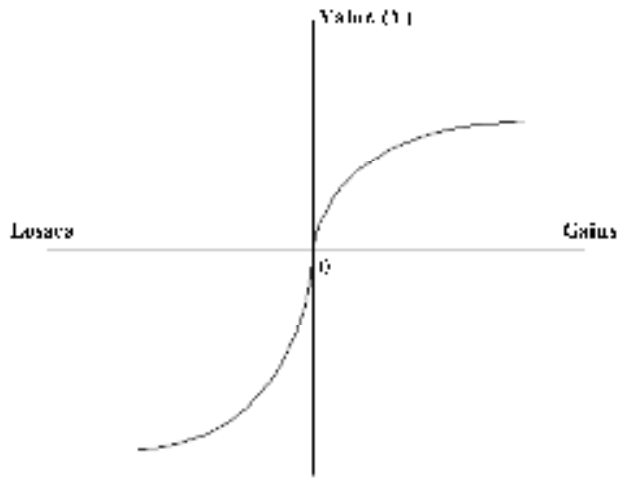

Fig. 1. Value Function

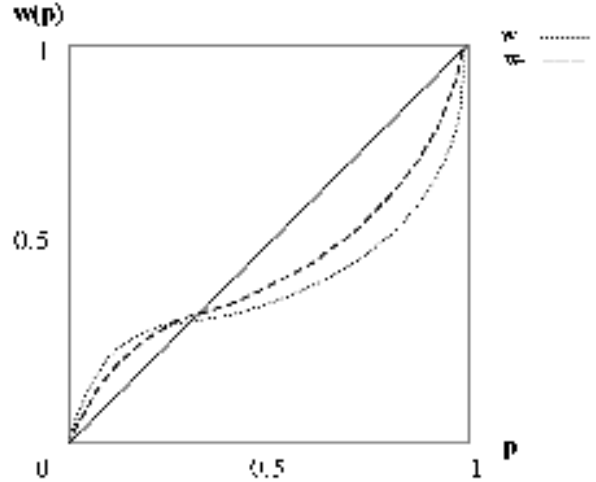

Fig. 2. Weighting Function

The weighting function of PT replaces the probability in the expected utility. Decision weight is the subjective judgment from decision maker according to probability, i.e. the psychological probability of decision maker. Fig. 2 shows the weighting function where $w(p)$ is monotonically increasing with probability $p$. The specific expression is shown as Eq. 2.

$$
w(p)= \begin{cases}w^{+}(p)=\frac{p^{\gamma}}{\left(p^{\gamma}+(1-p)^{\gamma}\right)^{\gamma}} & \\ w^{-}(p)=\frac{p^{\delta}}{\left(p^{\delta}+(1-p)^{\delta}\right)^{\delta}} & \end{cases}
$$

$w^{+}(\cdot)$ and $w^{-}(\cdot)$ denote the weighting functions for gains and losses, respectively and are monotonic and exhibit inverse S-shapes [17] based on median estimates of $\gamma$ and $\delta$ in Eq. 2 [12].

$$
U=\sum v(\Delta x) w(p)
$$

The value function $v(\Delta x)$ and the weighting function $w(p)$ are important components of PT, which influence the prospect $U$ of the decision maker as displayed in Eq. 3. Tversky and Kahneman once indicated that expected utility theory and prospect theory were indispensable, because rational model was accorded all the methodological privileges of a self-evident truth, a reasonable idealization, a tautology, and a null hypothesis, which could describe rational behavior, and PT could describe individual irrationalities in reality [21].

A Probe into the Dynamic Reference Point of Network Public Opinion Evolution. Under network, netizens are often affected by the surroundings, but each one has his psychological expectation that the incident develops in accordance with his judgments. This psychological expectation is internal reference point. But the reference point is not always static, that is, with the event developing, netizen's psychological expectation would also transfer, which is dynamic reference point. On one hand, the dynamic reference point is adjusted due to past opinion feedback; on the other hand, the reference point is updated as a result of receiving new information, for example, all participants continuously interact with neighbors over time, so that each one reexamines the status and the expectation. Thus, in order to reflect netizens' psychological variation better, the present study introduces dynamic reference point from prospect theory proposed by Kahneman and Tversky. Moreover, we construct a modified HK model on the purpose of finding more reasonable and consistent with the real evolution of the network public opinion.

\section{Modified Hegselmann-Krause Model}

Hegselmann-Krause model. In continuous public opinion dynamics, Hegselmann-Krause model [7] in recent years has aroused many scholars' attention and interest, of which rules are as follows. 
Let $N$ be the number of agents in the group under consideration and $N$ agents are represented as a node set $V=\{1,2, \ldots, N\}$, where the opinion of agent $i$ is within the continuous interval of $[0,1]$. Let $T$ be discrete time as $T=\{1,2, \ldots, t, \ldots\}$. So $x_{i}(t)$ denotes the opinion of agent $i$ at the time of $t$. In addition, we assume that all agents have the same confidence level $\varepsilon$. For another agent $j$, its opinion at time $t$ is expressed as $x_{j}(t)$, then the distance between agents $i$ and $j$ is $d_{i j}=\left|x_{i}(t)-x_{j}(t)\right|$. If $d_{i j}<\varepsilon$, agent $j$ is regarded as a neighbor of agent $i$.

An agent $i$ takes only those agents $j$ into account whose opinions differ from his own not more than a certain confidence level $\varepsilon$ and then updates or adjusts his opinion. Fixing an agent $i$ and an opinion profile $x=\left(x_{1}, \ldots, x_{n}\right)$ this set of agents is given by $I(i, x)=\left\{1 \leq j \leq n|| x_{i}-x_{j} \mid \leq \varepsilon\right\}$. Thus the model with bounded confidence is given by Eq. 4 .

$$
x_{i}(t+1)=\frac{1}{I} \sum_{j \in I(i, x(t))} x_{j}(t) \quad \text { for } t \in T .
$$

Next, we introduce dynamic reference point from PT on the basis of $\mathrm{HK}$ model to discuss public opinion evolution.

The proposed model related to dynamic preference point. Social network is formed through agents' interacting with others. From HK model mentioned above, it can be seen that at time $t$, agent $i$ chooses neighbors to communicate with according to some conditions (i.e. confidence level $\varepsilon$ ) and change his opinion following the corresponding principle (Eq. 4) which would be displayed at time $t+1$. Obviously, although HK model involved confidence level, and other external factors on the interaction rules were considered from existing researches [9, 22], few studies took individual psychological expectation - the internal factor - into consideration on public opinion formation. In general, when some event occurs at time $t$, agents who are concerned with the event hold preliminary opinions and form certain psychological expectation (psychological reference point) according to the event description. Then for the sake of knowing about more information, they immediately interact with neighbors following $\mathrm{HK}$ interaction principle. Until time $t+1$, having received the latest news, agents change their opinions; meanwhile, they compare acquired information (support vs. opposition) with his opinion at time $t$. If the interaction result at time $t+1$ is lower than his opinion at time $t$, it indicates that individual's evaluation of developing prospect is relatively high; conversely, the evaluation is slightly low. In either case, individual would respond accordingly, i.e. adjusting interaction result at time $t+1$ to the latest psychological point in the hope of evaluating the event objectively and comprehensively. In other words, in the entire process of public opinion evolution, individual's opinion is influenced by external neighbors and internal expectation. Therefore, this paper puts forward a modified HK model to explore how individual psychological reference point and external interaction simultaneously affect network public opinion evolution.

Individual Opinion Value Calculation. Firstly, an agent thinks of interaction result as the gain or loss relative to the reference point. The calculation of gain or loss value $\Delta x$ (the range of opinion change) that interaction opinion value is related to the reference point is shown as Eq. 5.

$$
\Delta x_{i}(t-1, t)=x_{i}(t)-x_{i}(t-1)=\frac{1}{I} \sum_{j \in I(i, x(t))} x_{j}(t-1)-\frac{1}{I} \sum_{j \in I(i, x(t))} x_{j}(t-2), t \geq 2 .
$$

From Eq. 5, $x_{i}(t-1)$ denotes individual's reference point at time $t ; \Delta x \geq 0$ represents that the opinion value of agent $i$ is lower than the one after interaction; When $\Delta x<0$, represents that the opinion value of agent $i$ is higher than the one after interaction. In order to calculate individual opinion value, suppose that $x_{i}$ as the opinion value of agent $i$, obeying random distribution at time $t=0$ in the interval $[-1,1]$. Agents interact with neighbors keeping to HK principle at time $t=1$. Thus individual opinion value at time $t=1$ can be calculated, as shown as Eq. 6 . 


$$
x_{i}(1)=\frac{1}{I} \sum_{j \in I(j, x(0))} x_{j}(0)
$$

Since agents cannot grasp all information immediately at initial stage of the event, there is no psychological expectation at the beginning, i.e. the initial reference point is assumed to be $U_{i}(0)=0$. Then individual's opinion value at time $t=1$ is calculated as Eq. 7 .

$$
v\left(\Delta x_{i}\right)=\left\{\begin{array}{ll}
v^{+}\left(\Delta x_{i}\right)=\left(x_{i}(1)-U_{i}(0)\right)^{\alpha}, & \text { if } x_{i}(1)-U_{i}(0) \geq 0 \\
v^{-}\left(\Delta x_{i}\right)=-\lambda\left[-\left(x_{i}(1)-U_{i}(0)\right)\right]^{\beta}, & \text { if } x_{i}(1)-U_{i}(0)<0
\end{array} .\right.
$$

In Eq. 7, the larger degree of parameters $\alpha$ and $\beta$, the greater degree of concavity and convexity of the value function, which reflects the psychological behavior characteristics of individual's sensitivity to opinion variation. The parameter $\lambda>1$ demonstrates that agents are more sensitive to negative-opinion trend than the positive one. For simplifying the computation, this study takes the values as follows: $\alpha=0.89, \beta=0.92, \lambda=2.25[12,16]$.

Individual Opinion Change Weight Calculation. Thanks to interacting with others, it is clear that agents change their opinions at the next time more or less, so the opinion change is certain. Allowing for the probability of opinion change, we refer to PT and give the method of individual opinion change weight calculation as shown as Eq. 8.

$$
w\left(p_{i}(t)\right)=\left\{\begin{array}{l}
w^{+}\left(p_{i}\right)=\frac{p^{\gamma}}{\left(p^{\gamma}+(1-p)^{\gamma}\right)^{\gamma}} \\
w^{-}\left(p_{i}\right)=\frac{p^{\delta}}{\left(p^{\delta}+(1-p)^{\delta}\right)^{\delta}}
\end{array}\right.
$$

$w^{+}(\cdot)$ and $w^{-}(\cdot)$ denote nonlinear weighting functions for gains and losses, respectively. The parameters $\gamma$ and $\delta$, respectively, usually take the values as follows: $\gamma=0.61, \delta=0.69$ [12].

Individual Opinion Prospect Value Calculation. On the basis of individual Opinion Value and Opinion Change Weight, Opinion Prospect calculation is given as follows.

$$
U_{i}(t)=\sum v\left(\Delta x_{i}(t-1, t)\right) w\left(p_{i}(t)\right), \text { for } t \in T .
$$

It should be noted that at time $t=1, \Delta x_{i}=x_{i}(1)-U_{i}(0)$ (see Eq. 7); after time $t=2$ (i.e. $\left.t \geq 2\right), x_{i}(t)$ transform into $x_{i}(t)=\frac{1}{I} \sum_{j \in I(i, x(t-1))} U_{j}(t-1)$, where $U$ denotes individual opinion prospect value, then Eq. 7 is further written as Eq. 10:

$$
v\left(\Delta x_{i}\right)=\left\{\begin{array}{ll}
v^{+}\left(\Delta x_{i}\right)=\left(x_{i}(t)-U_{i}(t-1)\right)^{\alpha}, & \text { if } x_{i}(t)-U_{i}(t-1) \geq 0 \\
v^{-}\left(\Delta x_{i}\right)=-\lambda\left[-\left(x_{i}(t)-U_{i}(t-1)\right)\right]^{\beta}, & \text { if } x_{i}(t)-U_{i}(t-1)<0
\end{array} .\right.
$$

To sum up, the opinion prospect value of agent $i$ at time $t-1$ would become the current opinion value, also reference point at present. Then the process of re-interacting, forming a new prospect, and a new reference point contributes to reference point dynamics, thus public opinion evolving. The specific calculation steps are as follows:

Step 1. Combing Eq. 5, Eq. 6, Eq. 7 and Eq. 10, individual opinion value can be determined (see Eq. 11 and Eq. 12).

If time $t=1$, then 


$$
v\left(\Delta x_{i}\right)=\left\{\begin{array}{ll}
v^{+}\left(\Delta x_{i}\right)=\left[\left(\frac{1}{I} \sum_{j \in I(i, x(0))} x_{j}(0)\right)-U_{i}(0)\right]^{\alpha}, & \text { if }\left(\frac{1}{I} \sum_{j \in I(i, x(0))} x_{j}(0)\right)-U_{i}(0) \geq 0 \\
v^{-}\left(\Delta x_{i}\right)=-\lambda\left[-\left(\frac{1}{I} \sum_{j \in I(i, x(0))} x_{j}(0)\right)-U_{i}(0)\right]^{\beta}, & \text { if }\left(\frac{1}{I} \sum_{j \in I(i, x(0))} x_{j}(0)\right)-U_{i}(0)<0
\end{array} .\right.
$$

If time $t \geq 2$, then

$$
v\left(\Delta x_{i}\right)=\left\{\begin{array}{l}
v^{+}\left(\Delta x_{i}\right)=\left[\left(\frac{1}{I} \sum_{j \in I(i, x(t-1))} U_{j}(t-1)\right)-U_{i}(t-1)\right]^{\alpha}, \quad \text { if }\left(\frac{1}{I} \sum_{j \in I(i, x(t-1))} U_{j}(t-1)\right)-U_{i}(t-1) \geq 0 \\
v^{-}\left(\Delta x_{i}\right)=-\lambda\left[-\left(\frac{1}{I} \sum_{j \in I(i, x(t-1))} U_{j}(t-1)\right)-U_{i}(t-1)\right]^{\beta}, \text { if }\left(\frac{1}{I} \sum_{j \in I(i, x(t-1))} U_{j}(t-1)\right)-U_{i}(t-1)<0
\end{array} .\right.
$$

Step 2. Individual opinion change weight can be acquired according to Eq. 8 .

Step 3. Individual opinion prospect value can be calculated based on Eq. 9.

Step 4. Iterate Steps 1 to 3, that is, agents interact with neighbors continuously, then psychological reference point transfers over time, finally public opinion evolution forms.

\section{Simulation Results and Analysis}

The Choice of Simulation Platform. Currently there are many modeling tools for Agent-based, such as NetLogo, RePast, Swarm, etc. [23]. But we chose Netlogo as simulation platform, because it is well suited for modeling complex systems developing over time as we need exactly. Moreover, it has extensive documentation and tutorials that help simulate our proposed model.

The parameters of proposed model were set in NetLogo as follows: the number of agents $N=1000$, confidence level $\varepsilon=0.3 ; \alpha=0.89, \beta=0.92, \lambda=2.25, \gamma=0.61$ and $\delta=0.69$ with reference to PT [12, 16]. Considering differences in probability of changing one's opinion next time, we preferentially assumed that the probability $p$ of all agents changing opinion was fixed, i.e. all were homogeneous, and $p=0.5$.

Simulation results on modified HK model. The modified HK model was simulated on NetLogo (operating time: ticks $=200$ ) and the operating results are shown in Fig. 3, Fig. 4, Fig. 5, and Fig. 6.

In Fig. 3 we can see that agents' opinions fluctuate dynamically and seriously at first, but after a certain period of time, each individual opinion tends to change fluctuate stably. Fig. 4 shows the tendency of sum opinion, which is also relatively stable finally. Fig. 5 and Fig. 6 demonstrate the number of clusters and the mean of opinions, respectively. In short, at the beginning of incident outbreak, none could collect enough information so that public opinions are divergent obviously. With agents knowing about incident development by degrees, their opinions are inclined to be unified, forming a small number of opinion clusters.

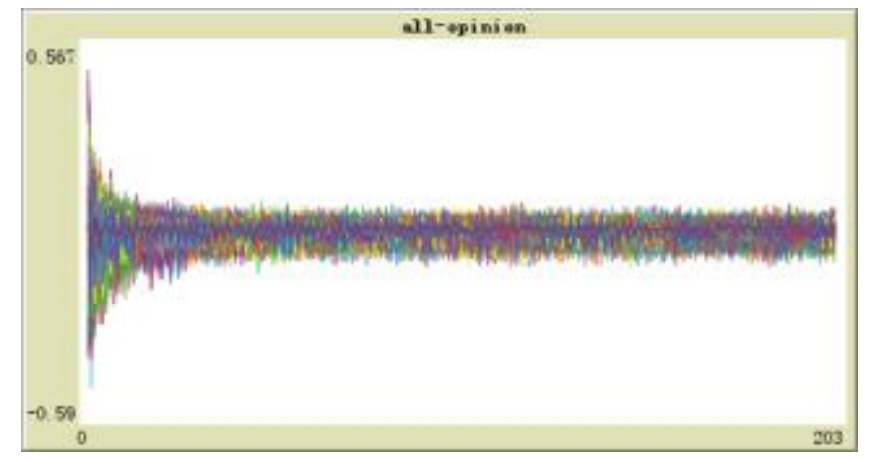

Fig. 3. Individual Opinion Variation

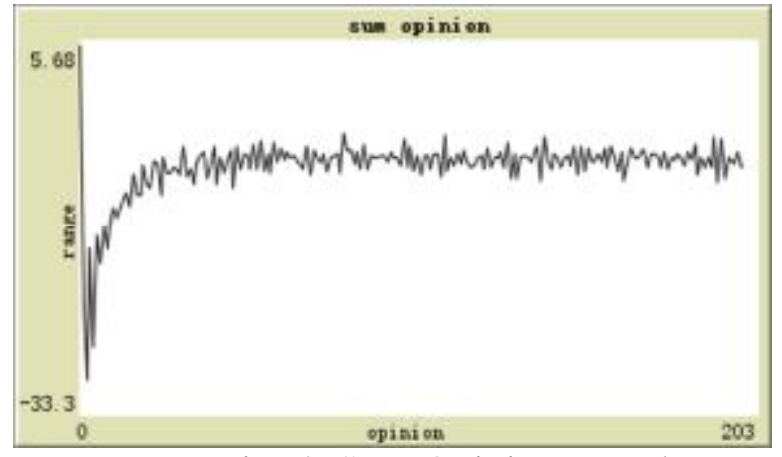

Fig. 4. Sum Opinion Trend 


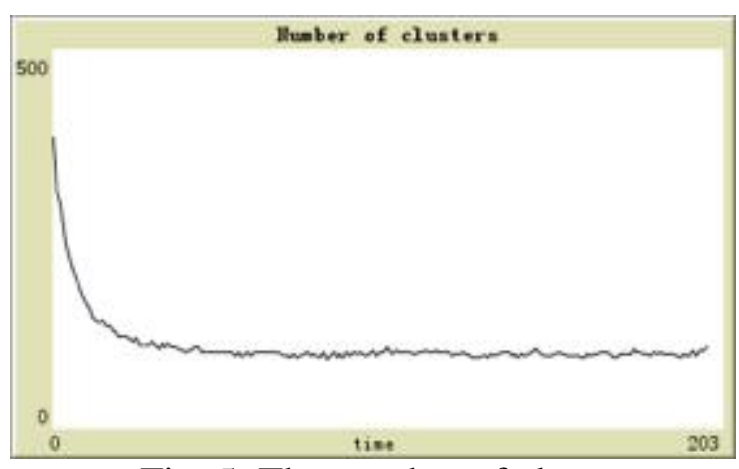

Fig. 5. The number of clusters

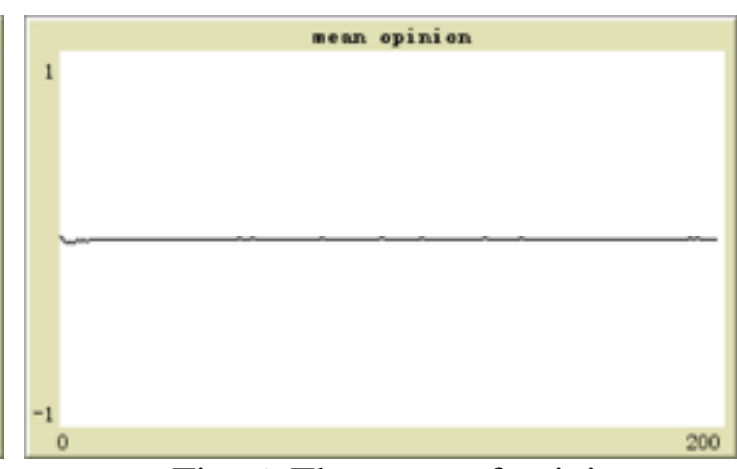

Fig. 6. The mean of opinions

The above simulation results indicate that the modified HK model reflects the volatility of individual opinion, revealing the instability under individual psychological expectation and interaction simultaneously. In fact, everyone is less likely to have the exactly same opinion, as no leaves are exactly the same in the world. Therefore, the proposed model demonstrates subtle differences in individual opinions and psychological expectations.

\section{Conclusions}

Simon has said that there is no completely rational human in real life [10]. Moreover, human's psychology (e.g. limited perception and ability to deal with information) affects decision-making and judgments greatly in reality. Contrast to existing studies that assume human as completely rationality, this paper defines agents under network public opinion as bounded rationality, draw into personal dynamic reference point (psychological expectation) and construct a new modified HK model based on Prospect Theory in order to analyze how dynamic reference point influence the evolution. Thanks to considering individual bounded rationality and the situation that psychological reference point is adjusted consistently in the evolution, the proposed model is more realistic than the traditional one. In addition, we provided government a direction on network management, that is, to strengthen netizens' psychological guidance. For example, related departments firstly take measures to divert netizens' attention, equivalent to adjust psychological reference point, in case of emergencies outbreak. However, the parameters used in model construction refer to the experimental data from Kahneman and Tversky which may be different from domestic group characteristics. In addition, we assumed that all agents were homogeneous in the simulation regardless of individual's different endowments which is our follow-up research in the future.

\section{Acknowledgements}

This work was financially supported by National Natural Science Foundation of China: "The Research of Crowd Simulation in the Process of Evolution of Network Public Opinion of Unexpected Event" (No.71273132) and National Natural Science Foundation of China: "Opinion Leader Identification and Policy Research Based on Sentiment Orientation Analysis” (No.71303111).

\section{References}

[1] Information on http://www.cnnic.net.cn/

[2] A. D. Mare, and V. Latora. International Journal of Modern Physics C, Vol. 18-9 (2007), pp. 1377-1395.

[3] D. Borra and T. Lorenzi, Zeitschrift Für Angewandte Mathematik Und Physik, Vol. 64-3 (2013), pp. 419-437.

[4] M. J. Hornsey, L. Blackwood, W. Louis, K. Fielding, K. Mavor, and T. Morton, et al. Journal of Applied Social Psychology, Vol. 36-7 (2010), pp. 1701-1722. 
[5] Xiaoyang Cao, Shujin Cao, and Guihong Chen (In Chinese). Information Science, Vol. 28-2 (2010), pp. 231-234.

[6] Min Lin (In Chinese). Internet Public Opinion: Research on Affecting Factors and Their Mechanism. Ph.D. dissertation, Zhejiang University, China, (2013).

[7] R. Hegselmann, and U. Krause. Journal of Artificial Societies \& Social Simulation, Vol. 5-3 (2002), pp. 1-33.

[8] J. A. Hołyst, K. Kacperski, and F. Schweitzer. Physica A Statistical Mechanics \& Its Applications, Vol. 285-1 (2000), pp. 199-210.

[9] Guiyuan Fu, Weidong Zhang, and Zhijun Li. Physica A Statistical Mechanics \& Its Applications, Vol. 419 (2015), pp. 558-565.

[10]H. A. Simon. The American Economic Review, Vol. 69-4 (1979), pp. 493-513.

[11]D. Kahneman, and A. Tversky. Econometrica, Vol. 47-2 (1979), pp. 263-292.

[12] A. Tversky, and D. Kahneman. Journal of Risk and Uncertainty, Vol. 5-4 (1992), pp. 297-323.

[13] R. Barkan, and J. R. Busemeyer. Journal of Behavioral Decision Making, Vol. 16-4 (2003), pp. 235-255.

[14] A. Hack, and F. V. Bieberstein. Review of Managerial Science, Vol. 9-1 (2015), pp.33-59.

[15] T. K. Tarnanidis, N. Owusufrimpong, and R. Marciniak. Marketing Review, Vol. 10-3 (2010), pp. 269-286.

[16]Liang Wang, Zixin Zhang, and Yingming Wang. Expert Systems with Applications, Vol. 42-23 (2015), pp. 9379-9388.

[17] Yang Liu, Zhiping Fan, and Yao Zhang. Computers \& Operations Research, Vol. 42-2 (2014), pp. 75-82.

[18] C. Paraschiv, and R. Chenavaz. Housing Studies, Vol. 26-3 (2011), pp. 329-352.

[19] H. Bleichrodt, U.Schmidt, and H. Zank. Management Science, Vol. 55-5 (2009), pp. 863-873.

[20] U. Schmidt, and H. Zank. Journal of Risk \& Uncertainty, Vol. 45-2 (2012), pp. 97-113.

[21] A. Tversky, and D. Kahneman. The Journal of Business, Vol. 59-4 (1986), pp. 251-278.

[22] Yanan Zhang, Jianjia He (In Chinese). Computer Engineering and Applications, Vol. 52-22 (2016), pp. 68-74.

[23] D. A. Robertson. Academy of Management Learning \& Education, Vol. 4-4 (2005), pp. 524-527. 